\title{
Balancing innovation and medical device regulation: the case of modern metal-on-metal hip replacements
}

This article was published in the following Dove Press journal:

Medical Devices: Evidence and Research

9 August 2016

Number of times this article has been viewed

\author{
Jason J Howard ${ }^{1,2}$ \\ 'Division of Orthopedic Surgery, \\ Department of Surgery, Sidra Medical \\ and Research Center, ${ }^{2}$ Department \\ of Orthopedic Surgery, Weill Cornell \\ Medical College, Doha, Qatar
}

\begin{abstract}
Due to problems with wear particle generation and subsequent loosening using conventional metal-on-polyethylene total hip replacements, there has been a shift toward alternative bearing systems, including metal-on-metal (MoM), for younger, more active patients with degenerative joint disease. Based on positive results from early short-term clinical studies, MoM hip replacements were readily adopted by orthopedic surgeons with thousands being implanted worldwide over the past decade. Unacceptably high revision rates reported by two national joint registries called into question the rigorousness of the regulatory approval process for these implants, particularly with respect to premarket data requirements to prove safety, effectiveness, and the appropriateness of the regulatory pathway chosen. The purpose of this review was to investigate the balance between facilitating the introduction of new medical technologies and the need to ensure safety and effectiveness through comprehensive regulatory assessment. The case of MoM hip replacement devices was used to frame the investigation and subsequent discussions. The regulatory approval processes and post-market surveillance requirements associated with three common MoM hip replacements (two resurfacings: the Birmingham and articular surface replacement and the articular surface replacement XL total hip replacement) were investigated. With respect to modern MoM hip replacement devices, the balance between facilitating the introduction of these new medical technologies and the need to ensure safety and effectiveness through comprehensive regulatory assessment was not achieved. The lessons learned from these experiences have application beyond joint replacements to the introduction of new medical technologies in general, particularly for those who have a significant potential for harm. In this regard, a series of recommendations have been developed to contribute to the evolution of the medical device regulatory process.
\end{abstract}

Keywords: medical devices, regulation, safety and effectiveness, metal-on-metal hip replacement

\section{Introduction}

Total hip replacement (THR) is one of the most successful medical advancements of the 20th century. The burden of illness for which these devices are indicated is vast, with more than 300,000 primary or revision hip replacement procedures performed in 2010 in the US alone. ${ }^{1}$ The most common indication for primary THR is for the reduction in pain secondary to joint degeneration associated with osteoarthritis, but numerous other diagnoses that result in the eventual destruction of the hip joint can also be reliably treated by THR. Since the advent of the concepts of "low-friction arthroplasty" introduced by the British surgeon Sir John Charnley in the early 1960s, the evolution of the THR has led to a device that can successfully reduce or eliminate
Correspondence: Jason J Howard

Division of Orthopedic Surgery,

Department of Surgery, Sidra Medical and

Research Center, PO Box 26999, Doha,

Qatar

Tel +974 44042274

Fax +97444041969

Email jason.howard@me.com 
the pain associated with hip arthritis for most cases. ${ }^{2}$ The most successful materials used in a THR bearing couple have traditionally been cobalt-chrome-molybdenum ( $\mathrm{CoCrMb})$ on ultra-high molecular weight polyethylene, with $>95 \%$ device survival at 10 years for this simple "metal-onplastic" concept. Despite its success, revision rates for this combination have been relatively high for the more active, younger patient population, with proportional increases in polyethylene wear that has been linked to the development of aseptic loosening. The increased demands of this younger patient demographic has fueled the drive to develop products with expanding indications and the potential for even better results, leading to a surge in new THR designs utilizing alternate bearing solutions. ${ }^{3,4}$ As such, previously abandoned bearing couples such as $\mathrm{CoCrMb}$ on $\mathrm{CoCrMb}$, commonly known as metal-on-metal (MoM), have experienced a resurgence in popularity over the past 2 decades, with the goal to design low wear, long-lasting THR solutions that mitigate the issues relating to high patient demand and early device failure.

MoM bearing surfaces have been used for decades with hip resurfacing procedures reported as early as $1948 .{ }^{4}$ There are two main types: 1) resurfacing devices that partially replace the femoral head, which is cemented into the neck of the femur and 2) THR devices with standard femoral stems and modular femoral head components. The attraction toward MoM implants was because of the fact that they were reported to generate less volumetric wear as compared to metal-on-polyethylene (MoP). These designs have historically been marketed alongside Charnley's THR, with some of the early MoM devices (eg, the McKee-Farrar) demonstrating similar long-term survival to this "gold standard". 5 Despite this, MoM devices eventually fell out of favor due to increased problems with premature wear, implant failure, and loosening, which, at the time, were blamed on unreliable manufacturing techniques, poor machining tolerances, and inadequate surgical instrumentation. In addition, concerns regarding the potential for carcinogenesis secondary to metal ion-induced DNA damage had put a damper on the enthusiasm for this technology.

Despite these previous concerns, it was felt that a new understanding of joint tribology and modern-day manufacturing techniques would overcome these problems so that the potential advantages of the MoM bearing couple could be realized. Since the incidence of osteolysis and subsequent implant loosening was linked to wear rates, it was expected that MoM designs would fare better than MoP, particularly for young patients with high activity levels. ${ }^{6}$ As such, a resurgence of interest in MoM device development ensued during the late 1980s, with implant companies and key orthopedic surgeon thought leaders moving to redesign and repopularize the technology. Indeed, during this time, there was definite success with the Metasul MoM articulation (Sulzer Inc., Winterthur, Switzerland) with revision rates at 10 years comparable to MoP at $\sim 7 \%$, serving to further stoke the interest in MoM technology.?

Based on the Metasul experience, and positive results from early short-term clinical studies involving newer implants, MoM devices were readily adopted by orthopedic surgeons to serve the rapidly expanding younger patient demographic with thousands being implanted worldwide over the past decade. ${ }^{8,9}$ Unfortunately, results from national joint registries in the UK and Australia subsequently revealed that revision rates for this class of devices were unacceptably high, up to four times the MoP "gold standard" THR. ${ }^{10,11}$ These findings call into question the rigorousness of the regulatory approval process for these implants, particularly with respect to premarket data requirements to prove safety, effectiveness, and the appropriateness of the regulatory pathway chosen (eg, 510[k] vs premarket approval [PMA] and choice of predicate devices).

The purpose of this review was to investigate the balance between facilitating the introduction of new medical technologies - with the potential for significant associated clinical and economic benefits - and the need to ensure safety and effectiveness through comprehensive regulatory assessment. The MoM class of hip replacements is analyzed as an example of a potential failure to maintain this balance with a view to identify pitfalls in the regulatory process and develop a series of recommendations that could be applied for similar technologies in the future. Implications for the companies involved will also be explored including: 1) their responsibilities to show cost effectiveness of the devices that they introduce, 2) the financial implications associated with such device failures, and 3) the due diligence required when introducing new technologies.

\section{MoM devices and regulation}

The reintroduction of the MoM THRs in the early 1990s was readily embraced despite a significant lack of long-term data to substantiate the hype surrounding the concept. In addition to low wear rates, other purported benefits of the bearing couple included: 1) less resection of femoral bone during implantation, 2) improved joint range of motion and joint stability associated with the large diameter bearing surfaces possible with MoM, and 3) improved activity level. ${ }^{6}$ 
Interestingly, over time, it was discovered that, as compared to MoP devices, no significant differences in these benefits were found between the device classes. In fact, in 2010, the National Joint Registry in the UK - which maintains a nationwide database of the outcomes of THRs implanted in the country - identified several problems associated with the use of MoM implants including: 1) severe metallosis reaction with soft tissue and bony destruction, 2) a high incidence of femoral neck fracture, 3) significantly higher revision rates in women, and 4) a very high failure rate in one particular implant system known as the articular surface replacement (ASR). ${ }^{11}$

A subsequent study of 31,932 patients, using data from the same UK registry, showed revision rates of MoM THRs to be almost double that of MoP THRs (5.2\% vs $2.8 \%)$. It was also found that women fared poorly as compared to men with revision rates of $8.5 \%$ and $3.6 \%$ at 5 years, respectively. In males, MoM resurfacing implants with large femoral heads fared much better, with revision rates comparable to MoP devices. ${ }^{11}$ This study became the catalyst for the eventual recall of one of the most popular modern MoM devices and the gradual erosion of the MoM market. Two quotes from the "Discussion" section of this study are worth repeating:

1. We need to learn the lessons from resurfacing and stemmed MoM when introducing these new technologies.

2. Regulators need to balance the need for innovation with the acceptability of risk.

To both encourage innovation and prevent patient harm, regulatory bodies must achieve a tricky balance. In the case of the reintroduction of MoM implants, this balance seems to have tipped too far in one direction, resulting in thousands of needless implant revisions and an erosion of public confidence in the regulatory system. ${ }^{9}$ In preparation for a detailed discussion regarding the regulatory pathways taken by three common MoM devices, a brief overview of the US regulatory requirements is given in the following sections.

\section{Brief overview of medical device regulation pathways}

The Food and Drug Administration (FDA), as the regulatory body governing the approval of medical devices in the US, has a mandate to ensure the safety and effectiveness of class II and III devices. Class II devices are higher risk devices but not life threatening and most are not exempt from general (ie, assurance of safety and effectiveness) and specific controls (ie, requirements for special labeling, performance standards, and post-market surveillance). Class III devices are those that support life, can be life-threatening, or have the potential for a significant risk of injury or illness associated. With respect to requirements for safety, the FDA must judge that the potential clinical benefits outweigh the potential risks when the device is used as intended, in a specified treatment setting, with adequate warnings against unsafe use. To prove effectiveness, data supplied to the FDA by the applicant must show that clinically significant results have been achieved. These requirements are both met through the provision of valid scientific evidence that can take many forms depending on the regulatory pathway taken. ${ }^{12}$

Most class II devices will be suitable for a $510(\mathrm{k})$ pathway. Under the $510(\mathrm{k})$, the device in question must be found to be substantially equivalent to a device that has been previously cleared by the FDA. Substantial equivalence is demonstrated when the device of interest is similar to a device(s) that has been: 1) legally marketed prior to 1976 (called a "pre-amendment" device) but not requiring a PMA, 2) reclassified from class III to II or I, or 3) previously found to be substantially equivalent through the $510(\mathrm{k})$ process. This device is also known as a predicate. To qualify as a predicate, the device should have the same intended use and technological characteristics, in addition to being safe and effective. This is an important point that will be revisited in subsequent discussions pertaining to the appropriateness of chosen pathway for MoM devices. Even if the device has different technological characteristics than the predicate, it can still be deemed substantially equivalent if it is as least as safe and effective as that device. Most MoM devices have applied for or achieved FDA approval via this pathway. ${ }^{12}$

Class III devices require a PMA pathway for regulatory clearance, and, like the $510(\mathrm{k})$ pathway, supporting data are needed to satisfy safety and effectiveness requirements for the device in question. In the $510(\mathrm{k})$ process, data to prove safety and effectiveness may or may not require clinical data (eg, hip simulator data are often enough for many THR applications), whereby the PMA has an absolute requirement for clinical data, usually in the form of clinical trials. The PMA is a much more stringent pathway which typically has high financial and temporal costs associated as compared to the $510(\mathrm{k}) .^{12}$

In most instances, companies will seek the $510(\mathrm{k})$ as a quicker and less costly means of getting their device to market. For this newest generation of implants, MoM THRs were found to be similar enough to identified predicates for approval under the $510(\mathrm{k})$ process. MoM resurfacing devices 
did not satisfy the substantial equivalence requirement and as such needed to follow the PMA pathway. The following sections summarize the regulatory journeys of three important MoM devices: the Birmingham hip resurfacing (BHR) system (Smith \& Nephew, Memphis, TN, USA), the ASR hip resurfacing replacement, and the ASR XL THR (DePuy, Warsaw, IN, USA).

\section{Birmingham hip resurfacing}

The BHR device led the resurgence of interest in MoM hip replacements after being introduced by Midlands Medical Technology in $1997 .{ }^{13}$ The BHR is a highly polished, dual radius MoM bearing couple which is said to promote ideal joint lubrication resulting in very low wear rates. ${ }^{14}$ This device was buoyed by several successful early short-term studies and became immensely popular in the UK. As such, the BHR was subsequently acquired by Smith \& Nephew, with a view to market the device globally.

When the BHR was being evaluated for regulatory approval in the European Union (EU), it was classified as a class IIb device, which meant it was considered medium risk and required premarket data only in the forms of laboratory work and clinical literature reviews. There was no obligation to conduct a clinical trial. ${ }^{10}$ Subsequently, the BHR was approved and in use in the EU (and other countries) from 1997. Smith \& Nephew subsequently applied for regulatory approval via the FDA. Unlike the European experience, the FDA considered resurfacing a new technique without a suitable predicate to prove substantial equivalence under a $510(\mathrm{k})$ pathway. As such, the BHR had to apply via the more stringent PMA pathway with initial filing on July 19, 2004. ${ }^{15}$

To obtain PMA regulatory clearance, the company was required to prove safety and effectiveness by providing nonclinical laboratory studies (including biocompatibility, materials testing, kinematic analysis, and wear simulator studies) and clinical investigations data. The main clinical data used to support the safety and effectiveness requirements of the application were a large series of 2,385 hips by a single investigator (who also happened to be the inventor of the BHR, Derek McMinn). ${ }^{16}$ The metrics collected included device survivorship, radiographic data, pain and function, and patient satisfaction. Additional unpublished data on 3,374 hips performed by 140 surgeons and published reports documenting the experience of other centers completed the requirements. By sheer volume and heterogeneity of the clinical series included, it would seem that these data would be appropriate to support the application.
Of the patients enrolled in the "Overall McMinn Cohort", device survivorship of $98.5 \%$ for the 546 hips that were followed up to 5 years was demonstrated which, in addition to favorable bench-testing and biocompatibility studies, led the FDA to the conclusion that "the applicant has provided an adequate device description and the preclinical testing information provided a reasonable assurance of device safety". ${ }^{16}$ There was, however, concern from some panel members that the prevailing clinical evidence was from a single surgeon and that "it did not contain data on the variability of the use of the device at various centers". Despite this discussion, the FDA's Orthopaedic and Rehabilitation Devices Panel (ie, the Panel) issued the recommendation to approve the PMA application with only three of five Panel members in favor.

Interestingly, only months after the FDA approval had been issued, a randomized controlled trial comparing the BHR with a time-tested MoP THR device (Exeter; Stryker) had to be stopped prematurely due to the high number of MoM device failures requiring revision (73\% required revision at an average of 8.5 years). ${ }^{17}$ By comparison, the National Institute for Health and Care Excellence (NICE) - the major health technology assessment body in the UK - set its benchmark revision rate as $<10 \%$ at 10 years post implant. ${ }^{18}$ The results of this study were not available at the time of the FDA assessment and eventual approval recommendation that occurred on September 8, 2005. ${ }^{16}$ This study highlights one of the major issues with proving safety and effectiveness for joint replacement devices: failures occur over many years such that short-term cohorts are often inadequate to guide recommendations for regulatory approval.

To its credit, however, the Panel did apply some conditions on the BHR PMA approval, which included the continuation of the main clinical study post approval to the 10 -year mark ensuring an adequate sample size based on sound statistical analysis. An assessment of the post-market surveillance requirements is discussed in the following sections.

\section{ASR hip resurfacing system}

Faced with the pressure to compete with the increasingly popular Birmingham hip, DePuy set out to develop an MoM resurfacing device of their own. Like the BHR, the ASR hip resurfacing system was deemed to be a new technique requiring a PMA pathway. This device had already been in use in the UK for several years prior to DePuy's application to the FDA. In August 2009, the FDA sent a confidential letter to DePuy informing the company that its ASR resurfacing device failed 
to meet PMA requirements and stating that the safety and effectiveness data the company supplied with their application were inadequate. Despite this, the company continued to market the device in the UK (as it had always done since the device's introduction in 2003) and was entitled to do so. ${ }^{19}$

\section{ASR $X L$ total hip system}

In addition to its resurfacing cousin, DePuy developed an MoM device that was implanted more like a traditional THR. The ASR XL had the same articulation as the ASR hip resurfacing with the exception that its large modular cobaltchrome head was impacted onto the trunnion of a femoral stem that was either press-fit or cemented into the femoral canal. To facilitate the use of existing femoral stems designed to accept much smaller heads, a metal adaptor sleeve was utilized which later turned out to be a significant source of metal debris generation. ${ }^{20}$ The regulatory approval process that the ASR XL followed was a much different pathway than that of the BHR system.

MoM THR devices that were on the market prior to 1976 are classified as pre-amendment devices. Despite their device classification, "they have been regulated via the 510(k) pathway as class III 510(k) devices". ${ }^{21}$ As discussed, the ASR hip resurfacing system was never approved in the US as it failed to provide the FDA with enough safety and effectiveness evidence under the PMA pathway. However, DePuy's companion product - the ASR XL THR - was subsequently approved based on the $510(\mathrm{k})$ pathway. This $510(\mathrm{k})$ clearance focused on finding predicates that would support claims of substantial equivalence for three aspects of the ASR XL: porous ingrowth surfaces, the MoM articulation itself, and the use of large femoral head sizes.

The 510(k) summary for the DePuy ASR XL listed seven devices as predicates to prove substantial equivalence for each of the different parts of the implant system. ${ }^{22}$ For example, the acetabular component was based on three devices: two previously approved ASR cup systems and one from another company. The predicates for the femoral head component and adapter sleeve were also from DePuy but from two different implant systems (ASR and Ultima) developed in recent years. The femoral stem component for the ASR XL had two predicates, again both from recent years, including the Corail AMT Hip Prosthesis and the DePuy Tri-lock Bone Preservation Stem. Regarding the basis of substantial equivalence, DePuy's application stated that the "DePuy ASR XL Modular Acetabular Cup components described in this submission are, in our opinion, substantially equivalent to[...]previously cleared [devices]... based on similarities of design, material composition and intended use/indications for use". ${ }^{21}$ They added that this application represented a simple modification to the device which involved adding larger cup sizes and that no new issues regarding safety and effectiveness were raised as a result of their submission.

The ASR XL 510(k) submission was received on April 7, 2008, and approval was sent on July 2, 2008, a quick turnaround compared to the experience of the BHR's PMA. ${ }^{21}$ The 2-year road to regulatory approval for the BHR PMA, however, was based on 44 pages of safety and effectiveness supporting data (described in the "Birmingham hip resurfacing" section) compared to the three-page $510(\mathrm{k})$ application for the ASR. The costs associated with the two pathways were also significantly different with a $510(\mathrm{k})$ application costing in the thousands and the PMA in the hundreds of thousands of dollars. The difference in financial and temporal resources required for these two regulatory pathways provides a strong incentive for companies to identify predicate devices that support claims of substantial equivalence under the $510(\mathrm{k})$.

In the case of the ASR XL, substantial equivalence was based on multiple "split" predicates rather that one device that has the same intended use, technological characteristics, and safety and effectiveness background. The predicates themselves had previous regulatory clearance based on substantial equivalence to other previously cleared devices, and so on. Hence, for the ASR XL, approval was ultimately achieved based on an "ancestry" of predicate devices dating back $\sim 50$ years. ${ }^{23}$ Looking back through the ancestry, clearance was essentially based on three main pre-amendment MoM devices: the McKee-Farrar system, the Ring prosthesis, and the Sivash prosthesis. Ironically, all three devices had previously been abandoned due to high revision rates, metallosis reactions, and concerns regarding metal ion exposure.

To prove substantial equivalence, the device in question is required to be at least as safe and effective as the chosen predicate device. ${ }^{12}$ It has been argued, however, that many of these pre-amendment predicates have never been formally assessed for safety and effectiveness and as such a finding of substantial equivalence under a $510(\mathrm{k})$ approval gives no assurance that the approved device meets these requirements. ${ }^{22}$ These concerns seemed to be substantiated when the 2009 report from the Australian Orthopaedic Association National Joint Replacement Registry reported the ASR XL revision rate to be $9.3 \%$ compared to $3.4 \%$ for MoP THRs at 5 years. ${ }^{24}$ In this case, the lack of premarket clinical data requirements for the $510(\mathrm{k})$ had disastrous results, as even a short-term clinical study with 2-year follow-up would have picked up the serious problems with the device. The problem 
was compounded by a lack of post-market surveillance data requirements for this case, which would have provided a safety net to catch device failures post approval had they been sought.

\section{Post-market surveillance}

Post-market surveillance provides a means for the FDA to obtain additional safety and effectiveness data following market approval for either the $510(\mathrm{k})$ or PMA pathways "when necessary to protect public health". ${ }^{25}$ A post-market surveillance system for medical devices should provide the following functions: 1) readily identifying poor performers, 2) communicating concerns regarding device performance (including benefits and risks) to relevant stakeholders, and 3) providing data that can be used to support regulatory approval for devices in future applications. ${ }^{26}$ Previously, the FDA could only order post-market surveillance of up to 36 months, but recent amendments to section 522 of the Federal Food, Drug, and Cosmetic Act have extended these periods.

Post-market surveillance can be ordered by the FDA to investigate: 1) problems identified in adverse event reports, 2) a change in where the device will be used, 3) potential long-term safety and effectiveness issues to supplement premarket data for implantables, and 4) the occurrence of serious adverse events associated with the device. ${ }^{25}$ Typically, a study plan is developed between the FDA and the device manufacturer, which usually follows the typical framework of a well-designed clinical trial. Failure to comply with the FDA order to complete a post-market surveillance study can result in the device being "misbranded" with associated penalties.

Although the FDA can order a post-market surveillance study for devices regardless of their regulatory pathway, typically the PMA pathway is more likely to require these data than devices cleared under a $510(\mathrm{k})$. For the BHR device, PMA was made contingent on substantial post-approval requirements including: the continuation of the main clinical trial out to 10 years, implementation of a multicenter component of the study to assess generalization of the procedure, implementation of a training program in the use of the device, and the provision of an analysis of adverse events and complaints received. ${ }^{27}$ By contrast, the ASR XL, approved via the $510(\mathrm{k})$ pathway, had no significant post-approval requirements other than the maintenance of general controls (including standard requirements such as annual registration, labeling, and good manufacturing practices). In 2011, however, due to the high failure rates for the MoM device class, the FDA had ordered manufacturers with currently marketed MoM devices to perform post-market surveillance studies. $^{28}$

\section{Repercussions: economic and clinical \\ Device companies and regulatory bodies}

As discussed in the "ASR hip resurfacing system" section, the FDA rejected DePuy's PMA application on the grounds that the company failed to show that the ASR hip resurfacing was safe and effective. By law, companies are not required to notify regulatory bodies in other regions when it fails approval in the US or another country. Thus, DePuy was within its rights to withhold information relating to its failure to achieve FDA approval under the PMA pathway. ${ }^{19}$ The practice of keeping the regulatory approval information confidential may have to be revisited, however, so that stakeholders, including surgeons, patients, and hospital administrators, can be aware of non-approvals and can make their own decisions as to how to go forward regarding the purchase and use of non-approved devices in their own region(s).

There are significant potential ramifications for companies in these circumstances over and above a direct financial impact. Device companies rely on surgeon "thought leaders" to be involved in investigational device exemption studies and to be early champions for their new technologies. In addition, for better or for worse, surgeons develop brand loyalty with device companies, which is very important to maintain a base market for the introduction of new devices through these end users. Withholding safety-related information by companies has the potential to cause irreparable damage to these relationships that can have lasting financial implications for the company. In addition to companies, cases such as these serve to sully the reputation of the FDA, which is charged to protect the public by ensuring the devices it approves for use are both safe and effective. As a result, there have been calls to "move away from the $510(\mathrm{k})$ process as soon as reasonably possible" in favor of a new medical regulatory framework that assures a more appropriate level of safety and effectiveness. ${ }^{29}$

\section{Clinical repercussions: the ASR recall}

In August 2010, DePuy recalled the ASR XL from the market because of UK registry data that showed revision rates of $13 \%$ at 5 years. ${ }^{30}$ This means that a substantial number of these patients will need to undergo repeat surgery which, in addition to causing significant discomfort, will likely increase 
the risk of postoperative complications including infection, bone loss, and dislocation. According to the field safety notice issued by DePuy, patients will need to be followed at least annually for 5 years with clinical and radiographic examinations. If the patient becomes symptomatic or if there are radiographic concerns, then metal ion blood levels should be measured and an MRI examination should be considered. If there are elevated levels and/or signs of tissue destruction, then the device should be revised. ${ }^{31}$ For patients who have not yet had failures, there is the stress of anticipating a problem as well as the concerns of metallosis and pseudotumor formation leading to revision. In these situations, the relationship between the surgeon and patient can also become strained, as the patient trusted him/her to make the best clinical decision with regard to their care.

Companies faced with device failures in the future would be best advised to provide timely and effective communication to patients, surgeons, and regulators and to institute device recalls at the earliest time possible based on the best available data. In addition, the intangible costs with respect to company reputation and surgeon loyalty in these cases will likely have longer lasting effects at an even higher cost.

\section{MoM devices and cost effectiveness}

In February 2014, the NICE performed a review of technology appraisal guidance including a cost effectiveness analysis and found that, "for all ages in both men and women, [traditional MoP] THR dominated resurfacing [MoM] arthroplasty over both the 10-year and lifetime time horizons". ${ }^{18}$ This analysis utilized Markov modeling incorporating costing with operational and revision data from national registries and randomized controlled trials to arrive at the conclusion that, on all fronts, traditional THR was significantly more cost-effective than resurfacing devices. The basis for this finding was related almost solely to the increased revision rates associated with resurfacing devices given the high costs associated as compared to primary nonrevision procedures.

In the recent past, the introduction of new medical technologies was often based on the wishes of the surgeon who would identify a device based on:(1) a belief that the new device would provide better outcomes, 2) patient demand for new technologies, 3) use in professional marketing, and 4) just being an "early adopter". For joint replacement procedures, these devices can amount to $30 \%-40 \%$ of the total cost for the procedure which can climb even higher with the adoption of more expensive bearing systems. ${ }^{32}$ Even a minor incremental "improvement" in a device's design can increase its price by $25 \%$ without evidence of concomitant improvement in patient outcome. As stakeholders become more aware of the implications of introducing untested technologies in the current cost-conscious health environment, this practice is being challenged.

As a device manufacturer, the case of the ASR, and modern MoM implants in general, should prompt reflection as to the due diligence required when acquiring new technologies for introduction into the market. The old model of "new is better... and more expensive" is changing in this new era of cost-effectiveness, where payers will choose which technologies are purchased based on evidence of the best clinical efficacy at the lowest cost. This shift in decisionmaking power from the surgeon to the administrator may be the catalyst for a concomitant shift in thinking which limits "chaotic innovation" in favor of tried and true technologies that offer lasting benefits with the least harm (Personal communication, Dr Michael Dunbar).

\section{Conclusion and recommendations}

Although tighter regulation and requirements for data on safety and effectiveness may cause a delay in market introduction for some new devices, the avoidance of fallout from poorly performing devices - including the erosion of public confidence, financial and reputational implications for companies (and surgeons), and most importantly the prevention of patient harm - should be embraced. Side-stepping tighter regulatory jurisdictions to bring medical devices to market more quickly without due diligence to safety and effectiveness are unethical and fly in the face of the Hippocratic oath that states "first do no harm".

Clearly, in the case of certain modern MoM THR devices, the balance between facilitating the introduction of these new medical technologies and the need to ensure safety and effectiveness through comprehensive regulatory assessment was not achieved. The lessons learned from these experiences have application beyond joint replacements to the introduction of new medical technologies in general, particularly for those who have a significant potential for harm.

Based on the discussions raised in this article, the following recommendations have been developed.

\section{Recommendation I}

With respect to appropriateness of regulatory pathway, it is recommended that class III (higher risk) devices with the potential for harm, like MoM THRs, should be evaluated for market clearance via a PMA rather than the less stringent $510(\mathrm{k})$ pathway regardless of their pre-amendment status. In 
this regard, the FDA's recent proposal to have manufacturers of currently marketed MoM devices to submit PMA applications is supported. ${ }^{33}$

\section{Recommendation 2}

The Institute of Medicine has previously recommended to eliminate the $510(\mathrm{k})$ process citing that it was not "fit for purpose" with their main concern being that there was no guarantee that the predicate devices were safe and effective. ${ }^{28}$ This assertion was rooted in the lack of previous formal assessment for many of these pre-amendment devices. As such, in the absence of formal safety and effectiveness data, it is recommended that if the historical use of a proposed predicate was not indicative of a device that was safe and effective (eg, taken off the market for increased failures) then it should not be approved for use in substantial equivalence claims.

\section{Recommendation 3}

With respect to the use of multiple (ie, split) predicates, there may be too much potential for the resulting combined predicate to not truly represent the device under review. In addition, the safety and effectiveness background of the combined predicate cannot be assured in a technology space where even minor adjustments (making the ASR's cup bigger and more shallow) can have dramatic effects on device performance. ${ }^{26}$ As such, it is recommended that the practice of multiple predicates be eliminated, particularly for devices with the potential for serious harm.

\section{Recommendation 4}

As was seen in the case of the ASR XL, the FDA criteria for the ordering of post-market surveillance studies are likely not stringent enough. For devices with the potential for serious harm, it is recommended that a requirement for additional post-market studies be issued for an appropriate duration post approval. In the case of THRs, a minimum requirement of 10 years should be required in keeping with NICE revision benchmark data. ${ }^{17}$

\section{Recommendation 5}

In an Orthopedic and Rehabilitation Devices Advisory Panel Meeting concerning MoM implant systems in 2012, the adverse outcomes reported in post-market studies were discussed as possible evidence that "traditional nonclinical testing of MoM hip systems may not be as predictive of clinically relevant failure modes as with other devices". ${ }^{19}$ As such, it is recommended that, for devices with the potential for serious harm, a requirement for premarket clinical data - with appropriate duration of follow-up - be enforced in addition to standard laboratory and benchmark data requirements. This recommendation should apply to both the $510(\mathrm{k})$ and PMA pathways.

\section{Recommendation 6}

Given the potential for harm to patients when a company withholds regulatory approval information from stakeholders, it is recommended that regulatory approval results be communicated freely to global regulators, surgeons, and patients to allow for informed decision making. This move would also provide an opportunity for the regulator to request additional safety and effectiveness data. There are FDA activities relating to this recommendation ongoing currently. ${ }^{26}$

\section{Acknowledgment}

The author would like to thank Professor Jan Pietzch (Stanford Biodesign, Faculty of Bioengineering, Stanford University, Palo Alto, CA, USA) who critically reviewed the study proposal and advised on study direction.

\section{Disclosure}

The author reports no conflicts of interest in this work.

\section{References}

1. Kurtz S, Ong K, Lau E, Mowat F, Halpern M. Projections of primary and revision hip and knee arthroplasty in the United States from 2005 to 2030. J Bone Joint Surg Am. 2007;89(4):780-785.

2. Wroblewski BM, Taylor GW, Siney P. Charnley low-friction arthroplasty: 19 to 25-year results. Orthopedics. 1992;15(4):421-424.

3. Meier B [webpage on the Internet]. In Medicine, New Isn't Always Improved. The New York Times; 2011. Available from: http://www. nytimes.com/2011/06/26/health/26innovate.html?pagewanted=1\&_r=0. Accessed April 1, 2014

4. Howcroft D, Head M, Steele N. Bearing surfaces in the young patient: out with the old and in with the new? Curr Orthop. 2008;22(3):177-184.

5. Jacobsson SA, Djerf K, Wahlström O. Twenty-year results of McKee-Farrar versus Charnley prosthesis. Clin Orthop Relat Res. 1996;329(suppl):S60-S68.

6. Garbuz DS, Tanzer M, Greidanus NV, Masri BA, Duncan CP. Metalon-metal hip resurfacing versus large-diameter head metal-on-metal total hip arthroplasty: a randomized clinical trial. Clin Orthop Relat Res. 2010;468(2):318-325.

7. Eswaramoorthy V, Moonot P, Kalairajah Y, Biant LC, Field RE. The Metasul metal-on-metal articulation in primary total hip replacement: clinical and radiological results at ten years. J Bone Joint Surg Br. 2008; 90(10): 1278-1283.

8. Treacy RBC, McBryde CW, Pynsent PB. Birmingham hip resurfacing arthroplasty: a minimum follow-up of five years. J Bone Joint Surg Br. 2005;87(2):167-170.

9. Steffen RT, Pandit HP, Palan J, et al. The five-year results of the Birmingham hip resurfacing arthroplasty: an independent series. $J$ Bone Joint Surg Br. 2008;90(4):436-441.

10. Cohen D. Out of joint: the story of the ASR. BMJ. 2011;342:d2905. 
11. Smith AJ, Dieppe P, Howard PW, Blom AW, National Joint Registry for England and Wales. Failure rates of metal-on-metal hip resurfacings: analysis of data from the National Joint Registry for England and Wales. Lancet. 2012;380(9855):1759-1766.

12. Pietzch JB, Aquino LM, Yock PG, Pate-Cornell ME, Linehan JH. Review of U.S. medical device regulation. J Med Dev Trans ASME. 2007;1(4): 283-292.

13. Cutts S, Carter PB. Hip resurfacing: a technology reborn. Postgrad Med J. 2006;82(974):802-805.

14. FDA [webpage on the Internet]. Public Meeting Orthopedic and Rehabilitation Devices Panel of the Medical Devices Advisory Committee; 2012. Available from: http://www.fda.gov/downloads/ AdvisoryCommittees/CommitteesMeetingMaterials/MedicalDevices/ MedicalDevicesAdvisoryCommittee/OrthopaedicandRehabilitationDevicesPanel/UCM310225.pdf. Accessed April 1, 2014.

15. FDA [webpage on the Internet]. Center for Devices and Radiological Health PMA Approval Letter; 2006. Available from: http:// www.accessdata.fda.gov/scripts/cdrh/cfdocs/cftopic/pma/pma cfm?num=p04003. Accessed April 1, 2014.

16. FDA [webpage on the Internet]. Summary of Safety and Effectiveness Data: Birmingham Hip Resurfacing System. Available from: http://www.accessdata.fda.gov/scripts/cdrh/cfdocs/cftopic/pma/pma. cfm?num=p04003. Accessed April 1, 2014.

17. Howie DW, McGee MA, Costi K, Graves SE. Metal-on-metal resurfacing versus total hip replacement-the value of a randomized clinical trial. Orthop Clin North Am. 2005;36(2):195-201.

18. NICE [webpage on the Internet]. NICE technology appraisal guidance 304. Total hip replacement and resurfacing arthroplasty for end-stage arthritis of the hip; 2014. Available from: https://www.nice.org.uk/ guidance/ta304. Accessed April 1, 2014.

19. Meier B [webpage on the Internet]. Hip Implant U.S. Rejected Was Sold Overseas. The New York Times; 2012. Available from: http://www. nytimes.com/2012/02/15/business/hip-implant-the-fda-rejected-wasmarketed-abroad.html?_r=1\&hp=\&pagewanted=all. Accessed April $1,2014$.

20. Bernthal NM, Celestre PC, Stavrakis AI, Ludington JC, Oakes DA. Disappointing short-term results with the DePuy ASR XL metal-onmetal total hip arthroplasty. J Arthroplasty. 2012;27(4):539-544.

21. Orthopaedic and Rehabilitation Devices Advisory Panel [webpage on the Internet]. FDA Executive Summary Memorandum. Metal-onMetal Hip Implant System; 2012. Available from: http://www.fda gov/downloads/AdvisoryCommittees/CommitteesMeetingMaterials/ MedicalDevices/MedicalDevicesAdvisoryCommittee/OrthopaedicandRehabilitationDevicesPanel/UCM309302.pdf. Accessed April 1, 2014.
22. Center for Devices and Radiological Health (FDA) [webpage on the Internet]. 510(k) summary: DePuy ASR XL Modular Acetabular Cup System; 2008. Available from: http://www.accessdata.fda.gov/ cdrh_docs/pdf8/K080991.pdf. Accessed April 1, 2014.

23. Ardaugh BM, Graves SE, Redberg RF. The $510(\mathrm{k})$ ancestry of a metalon-metal hip implant. $N$ Engl J Med. 2013;368(2):97-100.

24. de Steiger RN, Hang JR, Miller LN, et al. Five-year results of the ASR XL acetabular system and the ASR hip resurfacing system. J Bone Joint Surg Am. 2011;93(24):2287-2293.

25. Center for Devices and Radiological Health [webpage on the Internet]. Procedures for Handling Section 522 Postmarket Surveillance Studies; 2011. Available from: http://www.fda.gov/downloads/MedicalDevices/ DeviceRegulationandGuidance/GuidanceDocuments/UCM268141.pdf. Accessed April 1, 2014.

26. Roehr B. FDA proposes tightening post-market oversight of medical devices. BMJ. 2012;345:e6140.

27. Center for Devices and Radiological Health [webpage on the Internet] FDA Approval Letter, PMA Application: Birmingham Hip Resurfacing System; 2006. Available from: http:/www.accessdata.fda.gov/ cdrh_docs/pdf4/p040033a.pdf. Accessed April 1, 2014.

28. FDA [webpage on the Internet]. Metal-on-Metal Hip Implants: FDA Activities. Available from: http://www.fda.gov/medicaldevices/ productsandmedicalprocedures/implantsandprosthetics/metalonmetalhipimplants/ucm241769.htm. Accessed April 1, 2014.

29. Institute of Medicine of the National Academies. Committee on the Public Health Effectiveness of the FDA 510(k) Clearance Process. Medical Devices and the Public's Health: The FDA 510(k) Clearance Process at 35 Years. Washington, DC: The National Academies Press; 2011:280.

30. FDA [webpage on the Internet]. Metal-on-Metal Hip Implants: Recalls. Available from: http://www.fda.gov/medicaldevices/productsandmedicalprocedures/implantsandprosthetics/metalonmetalhipimplants/ ucm241770.htm. Accessed April 1, 2014.

31. FDA [webpage on the Internet]. Class 2 Device Recall Depuy ASR Resurfacing Femoral Heads. Available from: http://www.accessdata. fda.gov/scripts/cdrh/cfdocs/cfres/res.cfm?id=96073. Accessed April $1,2014$.

32. Sculco TP. The economics of new age arthroplasty: can we afford it? Orthopaedics. 2013;1(1):19-20.

33. Federal Register [webpage on the Internet]. Effective Date of Requirement for Premarket Approval for Two Class III Preamendments Devices. Available from: https://www.federalregister.gov/ articles/2013/01/18/2013-01006/effective-date-of-requirement-forpremarket-approval-for-two-class-iii-preamendments-devices. Accessed April 1, 2014
Medical Devices: Evidence and Research

\section{Publish your work in this journal}

Medical Devices: Evidence and Research is an international, peerreviewed, open access journal that focuses on the evidence, technology, research, and expert opinion supporting the use and application of medical devices in the diagnosis, monitoring, treatment and management of clinical conditions and physiological processes. The identification of novel
Dovepress

devices and optimal use of existing devices which will lead to improved clinical outcomes and more effective patient management and safety is a key feature. The manuscript management system is completely online and includes a quick and fair peer-review system. Visit http://www. dovepress.com/testimonials.php to read real quotes from authors. 